\title{
Idrope Fetale non Immunologia e Gravidanze Gemellari
}

\author{
G. Noia', M. De Santis', L. Neri' ${ }^{1}$, A. Serraino ${ }^{2}$, S. Storti' ${ }^{2}$, M.L. Gozzo ${ }^{2}$, E. \\ Maniccia', A. Caruso' \\ Istituti di ${ }^{1}$ Clinica Ostetrica, ${ }^{2}$ Semeiotica Medica e ${ }^{3}$ Chimica Clinica dell'Università Cattolica \\ del Sacro Cuore, Roma, Italia
}

\begin{abstract}
Presso il Centro di Diagnosi Prenatale all'Università Cattolica del Sacro Cuore di Roma, sono state osservate 95 pazienti con gravidanza complicata da idrope fetale non immunologica. Nove pazienti presentavano una gravidanza gemellare. La sopravvivenza dei 95 feti osservati è stata del $\mathbf{2 7 . 7 \%}$. Nessun feto gemellare idropico è sopravvissuto. Nei 9 casi di gravidanza gemellare è stato possibile diagnosticare ecograficamente una placenta monocoriale in 6 casi e monoamniotica in 3 casi. La causa dell'idrope fetale è stata riferita in 3 casi a trasfusione feto-fetale; in 2 casi a cardiopatia fetale ed 1 caso a malformazione genito-urinaria e 3 casi di n.d.d. La morte del feto idropico è avvenuta tra la $21^{\text {a }}$ e la $35^{\text {a }}$ settimana di gravidanza. Dei feti gemellari non idropici la sopravvivenza è stata del $57.1 \%$ (4 su 7).

Otto delle gravidanze gemellari sono state osservate prima del 1988, epoca in cui nella nostra Istituzione, non era possibile un approccio diagnostico e terapeutico intravascolare mediante cordocentesi. Tale possibilità diagnostica e terapeutica può con molta probabilità migliorare la sopravvivenza dei feti idropici nelle gravidanze gemellari come avvenuta nelle gravidanze singole, dove è passata dal 25\% (1980-88) al 36.8\% (1988-93).
\end{abstract}

\section{(Presentato come poster)}

Per Corrispondenza: Dr. G. Noia, Policlinico Universitario “A. Gemelli ", Largo A. Gemelli 8, 00168 Roma, Italia. 